\title{
Desarrollo embrionario-larval del pez tropical Hemirhamphus brasiliensis (Beloniformes: Hemirhamphidae) a partir de huevos recolectados del medio natural
}

\author{
Jesús Rosas ${ }^{1}$, Ernesto Mata ${ }^{1}$, Aidé Velásquez ${ }^{2} \&$ Tomas Cabrera ${ }^{2}$
}

1. Instituto de Investigaciones Científicas. Universidad de Oriente Núcleo de Nueva Esparta, Boca del Río, Estado Nueva Esparta, Venezuela; rosas@ne.udo.edu.ve; matapico@hotmail.com

2. Escuela de Ciencias Aplicadas del Mar, Universidad de Oriente Núcleo de Nueva Esparta, Boca del Río, Estado Nueva Esparta,Venezuela; avelasquez@ne.udo.edu.ve; tom3171@telcel.net.ve

\author{
Recibido 09-V-2006. Corregido 30-VI-2008. Aceptado 31-VII-2008.
}

\begin{abstract}
Embrionary-larval development of the tropical fish Hemirhamphus brasiliensis (Beloniformes: Hemirhamphidae) from eggs collected in the wild. The embryo formation and larval development of Hemirhamphus brasiliensis Linnaeus, 1758 (Pisces: Hemirhamphidae) is described from morula stage eggs collected on Sargassum sp. Thalii in the field (1050'55.2" N y 6409'467” W). The eggs were spherical, 1923.54 $\pm 72.35 \mu \mathrm{m}$ diameter with several corionic filaments, and are striated. During the first $48 \mathrm{~h}$ the embryo developed cephalic vesicle, miomers, and a heart located on the external body surface, beating strongly and circulating colorless blood which became pigmented red later. Before hatching, the larva developed kidney, gut tract, liver and biliar vesicle, pectoral fins, four pairs of gill arches and the mouth. The larva hatched at $114 \mathrm{~h}$, the body was torpedo-shaped, yellow-green, with several dendriform melanophores; the pelvic fin was observed $72 \mathrm{~h}$ post hatching. At 240 hours the metamorphoses was completed. When the larvae hatched they could ingest Artemia metanauplii. Rev. Biol. Trop. 56 (3): 1449-1458. Epub 2008 September 30.
\end{abstract}

Key words: embryo, larvae, hatch, Hemirhamphus brasiliensis.

La familia Hemirhamphidae esta representada por especies pelágicas en aguas costeras y oceánicas. Entre sus representantes destacan Hemirhamphus brasiliensis, distribuida en el Atlántico, del lado occidental desde el norte de los Estados Unidos hasta Río de Janeiro, incluyendo todo el Golfo de México y las Antillas. En Venezuela es una especie frecuente $\mathrm{y}$ a veces muy abundante en la plataforma nororiental. Aunque es una especie comestible, su importancia fundamental radica en su utilización como carnada en la pesca deportiva (Cervigón 1991, McBride y Thurman 2003).

Las investigaciones referentes a $H$. brasiliensis están relacionadas con su abundancia en la pesquería industrial, riqueza íctica en lagunas costeras, dispersión de sus huevos sobre objetos flotantes (Castro et al. 2002), y recientemente sobre la biología reproductiva, maduración y frecuencia reproductiva (McBride y Thurman 2003). En el área de Caribe existe una gran variedad de especies de las cuales se desconoce su desarrollo embrionario y larval, debido a la importancia ecológica y pesquera de $H$. brasiliensis, la presente investigación tiene como objetivo describir la formación del embrión y el desarrollo larval, como una contribución al conocimiento de esta importante especie pelágica.

\section{MATERIALES Y MÉTODOS}

En julio del 2005 se realizaron salidas de campo entre las Islas de Margarita y Cubagua $\left(10^{\circ} 50^{\prime} 55.2^{\prime \prime} \mathrm{N}\right.$ y $64^{\circ} 09^{\prime} 467^{\prime}$ 'W) con el fin de 
revisar el desplazamiento de Sargassum sp., así como de otros objetos flotantes en donde muchos peces marinos depositan sus huevos, sobre todos los adhesivos o con filamentos coriónicos (McBride y Thurman 2003). Un día después de la fase de luna llena se detectaron numerosas masas de huevos en diferentes estados de desarrollo en arribazones de Sargassum sp., seleccionándose aquellos de la más reciente deposición, mediante el uso de una lupa de campo. Las porciones de talos de Sargassum sp. con los huevos adheridos fueron colocados en recipientes de $150 \mathrm{~L}$ con agua de mar, proveniente del lugar de recolección de los huevos, el agua fue previamente filtrada mediante una malla de $25 \mu \mathrm{m}$ y transportada al laboratorio del Instituto de Investigaciones Científicas en donde se procedió a su aclimatación a $28.5 \pm 0.4{ }^{\circ} \mathrm{C}$, paralelamente se detectó la fase de desarrollo y se midieron 50 huevos describiéndose los cambios embrionarios cada 24 h según el criterio de descripción larval señalado por Ahlstrom (1968) y Del Río et al. (2005). Las larvas nacidas fueron colocadas en tres recipientes de $150 \mathrm{~L}$ adicionándose durante las primeras $24 \mathrm{~h}$ rotíferos (Brachionus plicatilis) a $25 \mathrm{ind} / \mathrm{ml}$, copépodos (Apocyclops distans) a $12 \mathrm{ind} / \mathrm{ml}$ y la microalga Isochrysis galbana a $450.000 \mathrm{cel} / \mathrm{ml}$, posteriormente se adicionó metanauplios de Artemia de $48 \mathrm{~h}$ de nacidos $(4 \mathrm{ind} / \mathrm{ml})$ para el mantenimiento de las larvas. Cada cinco días se tomaron tres larvas para determinar aspectos morfometricos (longitud total, longitud notocordio, longitud aleta embrionaria, longitud horquilla, ancho de la larva), aspectos merísticos (longitud de la orbita, ancho de la orbita, longitud circulo orbitario, ancho circulo orbitario, longitud cuenca orbitaria y ancho cuenca orbitaria, hasta que se produjo su metamorfosis) y algunas observaciones sobre su fisiología.

\section{RESULTADOS}

\section{Aspectos taxonómicos}

Organogenesis: los huevos fueron localizados en estado de morula, eran esféricos transparentes con diámetro de $1923.54 \pm 72.35$ $\mu \mathrm{m}$, con estrías en la superficie y numerosos filamentos coriónicos (Fig. 1A), abundante vitelo y una gota lipídica. Transcurridas $24 \mathrm{~h}$ el embrión presentó ojos rodeados de numerosos melanóforos puntiformes; capsulas óticas a nivel medio del tronco, vesícula cefálica prominente. Los miomeros eran de forma circular y el cuerpo con secciones o bandas marrones (Fig. $1 \mathrm{~B}$ )

A las $48 \mathrm{~h}$, se observó los ojos pigmentados, así como la vesícula cefálica. El corazón (Fig. 1C) se ubicó en la parte posterior fuera del cuerpo embrionario, se desplegó la aleta pectoral izquierda, la aleta caudal presentó una forma globosa, en la parte media del tronco se observó una vesícula de rojo intenso (Fig. 1D). Todo el cuerpo se cubrió de melanóforos puntiforme y dendriforme hacia la parte interna del embrión, el cual se ubicó sobre el vitelo, el notocordio era transparente (Fig. 1E).

A las $72 \mathrm{~h}$ Las capsulas óticas se ubicaron por detrás de los ojos. Se observó presencia de aletas pectorales, la aleta caudal se observó encima de la región cefálica, la pigmentación del cuerpo no se alteró, la sangre se pigmentó de rojo (Fig. 1F). La vesícula en la región posterior se dividió en dos, estructuras similares se ubicaron por debajo de la vejiga de los gases. En este momento fue imposible cuantificar los miómeros.

A las $96 \mathrm{~h} \mathrm{La} \mathrm{boca} \mathrm{era} \mathrm{amplia,} \mathrm{se} \mathrm{observa-}$ ron arcos branquiales y opérculos desarrollados, ojos iridiscentes. Se observaron miómeros en paquetes compactos (Fig. 1G), la región cefálica blanca, aletas pectorales globosas con tres radios, aleta anal y dorsal desarrolladas, la aleta caudal de $475.47 \pm 1.68 \mu \mathrm{m}$ de largo con 12 radios y otros en desarrollo muy cerca del tronco. La región del complejo hipural con numerosos vasos sanguíneos. El corazón se desplazó al interior del cuerpo. El vitelo se redujo al $12 \%$ y estuvo unido al embrión en la región ventral en la intersección de la cabeza y el tronco. El tubo digestivo era largo, el estomago en desarrollo presentó un ciego en forma de pequeña lámina. Hígado en desarrollo con vesícula biliar. 

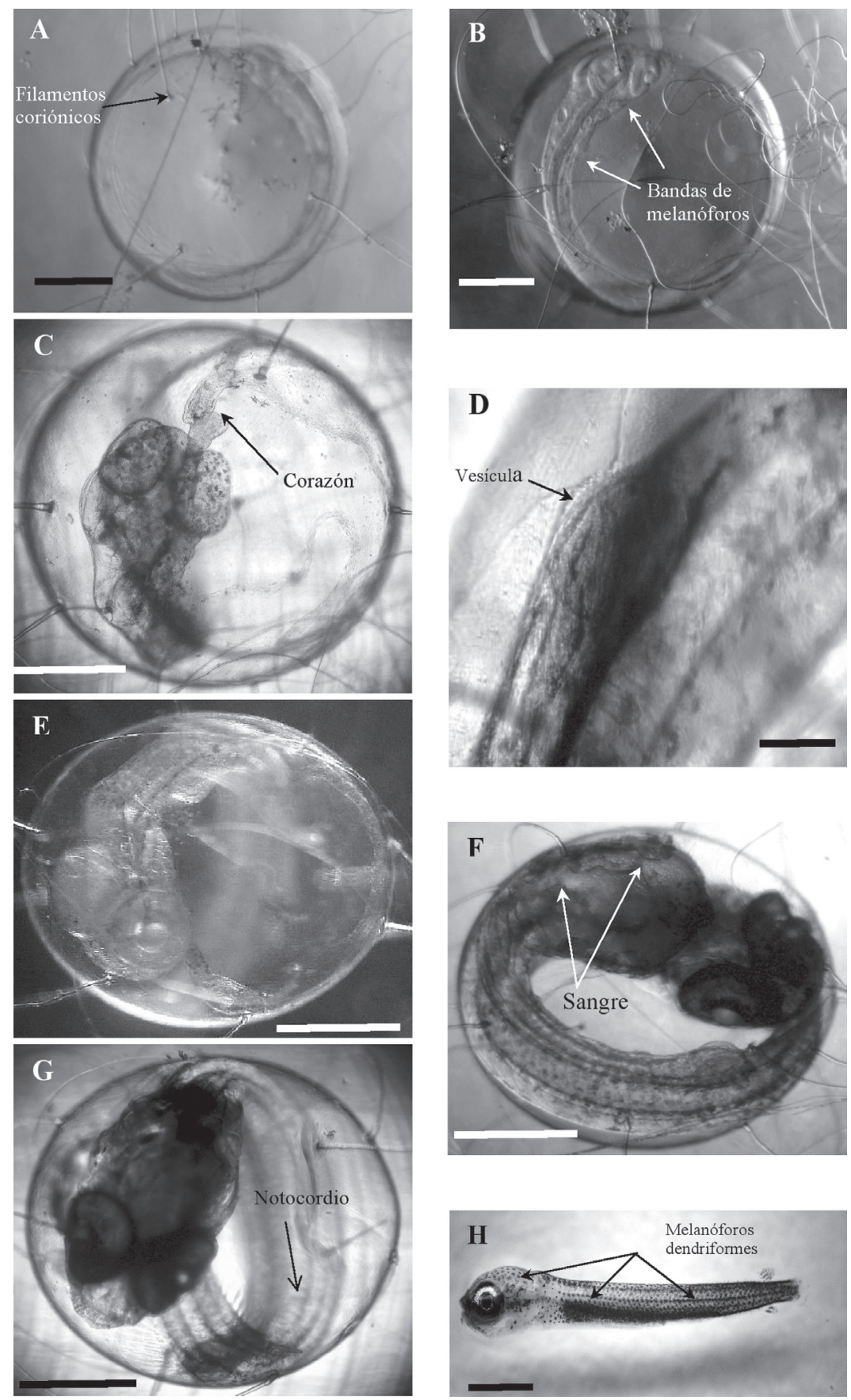

Fig. 1. Desarrollo de Hemiramphus brasiliensis. A) Filamentos coriónicos, B) Banda de melanóforos, C) Corazón, D) Vesícula, E) Distribución de melanóforos, F) Canales de circulación, G) Notocordio, H) Larva recién eclosionada. (Escala $500 \mu \mathrm{m})$.

Fig. 1. Embryonic development of Hemiramphus brasiliensis. A) Chorionic filament insertion, B) Melanophore bands, C) Heart, D) Vesicle, E) Melanophore distribution, F) Circulation channels, G) Notochord, H) Recently hatched larva. (Scale $500 \mu \mathrm{m})$. 
Desarrollo larval: las larvas eclosionaron a las 114 h (Fig. 1H), Poseían dientes así como tres pliegos (Fig. 2A) que parecían originarse en la parte superior de ambas mandíbulas y que llegaban a la boca. Poseían tres arcos branquiales y otro en desarrollo. El cuerpo era robusto en forma de torpedo, verde amarillo ampliamente pigmentado con melanóforos dendriformes negros, con aletas pectorales globosas con cuatro radios rudimentarios, aleta caudal con 16 radios, aletas anal y dorsal con 12 radios, ambas muy próximas a la caudal. Se observó la aleta anal con una línea negra a ambos lados (Fig. 2B). Región ventral del cuerpo transparente. El tracto digestivo con dos regiones diferenciadas: una región representada por una cavidad gástrica llena de vitelo y tapizada con células que originaron el estomago y la otra región que originó el intestino posterior con un par de ciegos en la unión con el intestino que era largo $(2.19 \pm 0.12 \mathrm{~mm})$ y finalizó en el ano sin apertura visible. Se observó el corazón, hígado y vesícula biliar.

A las $24 \mathrm{~h}$ de nacidas las larvas poseían, el cuerpo robusto ampliamente pigmentado con melanóforos dendriformes negros, boca amplia y pigmentada de negro, ojos grandes iridiscentes, opérculos abiertos protegiendo a tres pares de arcos branquiales hasta con 16 filamentos. La región cefálica se observó cubierta de melanóforos dendriformes negros, aleta dorsal transparente con 12 radios los cuatro últimos con un revestimiento negro, próximos a la aleta caudal que poseía 16 radios con tres segmentos, aleta anal con 13 radios los cuatro últimos recubiertos de negro. Las aletas pélvicas totalmente transparentes con seis radios. Internamente el estomago (Fig. 2C) era un tubo largo (3.12 \pm $0.09 \mathrm{~mm}$ ). Se detalló la vejiga de los gases.

A las $48 \mathrm{~h}$, de nacidas las larvas, el complejo hipural presentó un reborde de melanóforos negros, el número de radio permaneció sin alterar, el glóbulo de las aletas pectorales presentó un reborde negro. Internamente el tubo gastrointestinal midió $3.22 \pm 0.17 \mathrm{~mm}$ de largo y de $0.04 \pm 0.002 \mathrm{~mm}$ de ancho.

A las $72 \mathrm{~h}$, se observaron cuatro arcos branquiales en ambos lados de la región opercular de filamentos zigzagueados. La base de los radios de la dorsal con melanóforos, igual proceso ocurrió en la anal, mientras que en la caudal los melanóforos estaban dispersos en toda la aleta. El cuerpo presentó una línea en la región media, con melanóforos dispersos en la región ventral.

No se observaron cambios significativos en la estructura de las larvas a las $96 \mathrm{~h}$, salvo que la aleta caudal inició su proceso de orquillamiento, los radios de las aletas dorsal y anal estaban muy cerca de la caudal.

A las $120 \mathrm{~h}$, la aleta pectoral presentó seis radios (Fig. 2D) se inició el proceso de escamación, la región ventral con melanóforos dendriformes iridiscentes. La región dorsal totalmente cubierta de melanóforos, el resto de los caracteres anteriormente descritos permanecieron sin alteración.

A las $144 \mathrm{~h}$, las larvas presentaron la parte superior de la aleta caudal ligeramente más larga que la inferior, se pigmentó un nuevo radio de la aleta dorsal de negro y amarillo. A las $168 \mathrm{~h}$, se observaron seis segmentos en la aleta caudal, la región ventral totalmente coloreada de negro, la aleta dorsal (Fig. 2E) coloreada de amarillo con melanóforos ligeramente negros. Los arcos branquiales hasta con 14 branquiespinas.

A las $216 \mathrm{hrs}$, la característica más resaltante fue la aparición de escamas y se inició el alargamiento de la mandíbula inferior (Fig. 2F) de color rojo intenso que terminó de forma redondeada. Las larvas presentaron la aleta pélvica con los tres últimos radios de color negro, la zona superior de la línea lateral con pocas escamas, transparentes y algunos melanóforos dendriformes, la región ventral más oscura, plateada y cubierta de escamas hasta con seis líneas, la base de las aletas pectorales totalmente negras, ocho de los radios de la aleta dorsal (Fig. 2G) eran amarillos, unidos por una membrana negra, siete radios de igual características a la aleta anal, en ambos casos permanecieron muy próximos a la aleta caudal, cuya región inferior era más larga que la superior (Fig. 2H)

Al cumplir $240 \mathrm{~h}$, de nacidas las larvas alcanzaron la fase juvenil con el $80 \%$ del 

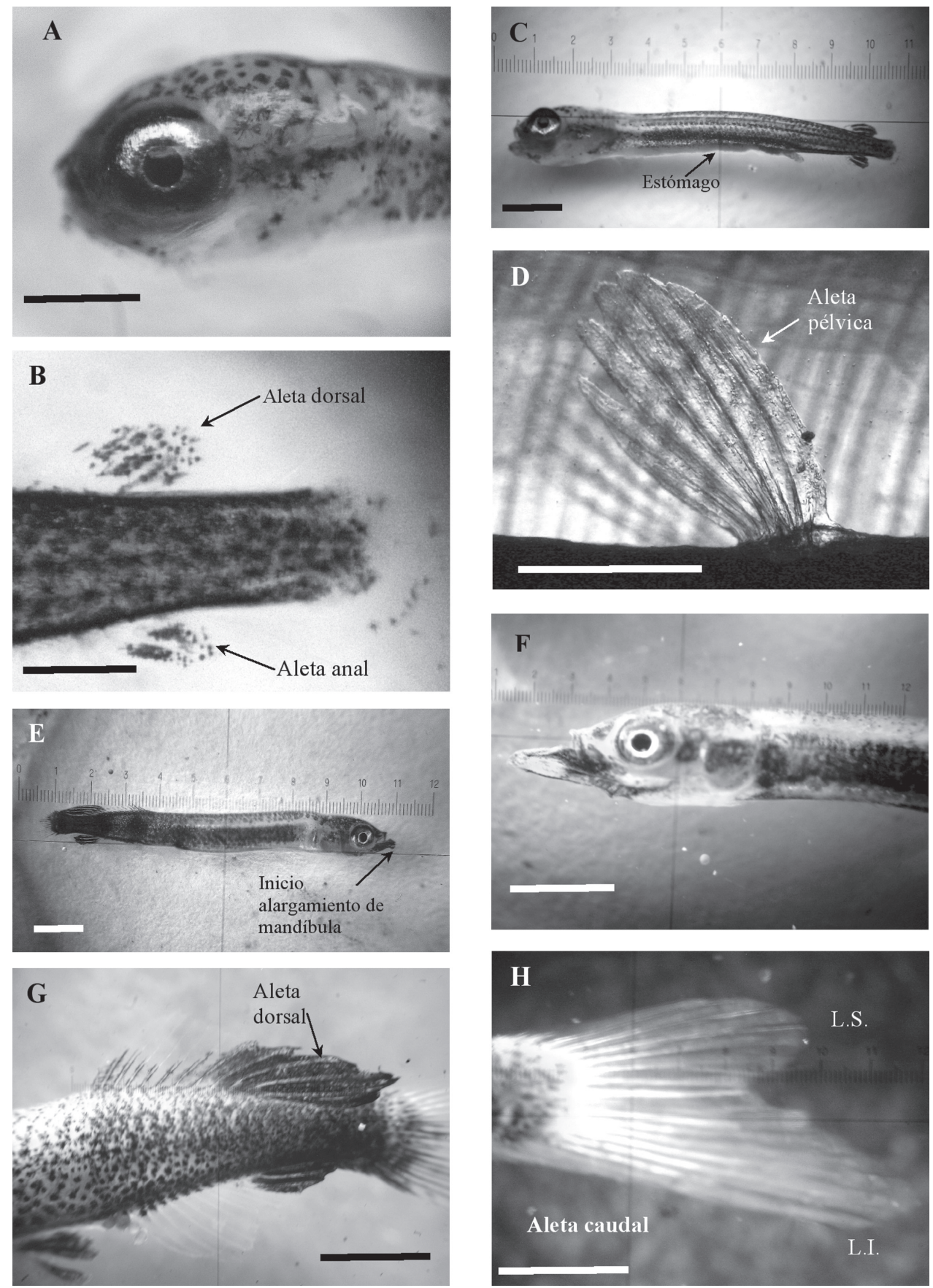

Fig. 2. Desarrollo larval de Hemiramphus brasiliensis. A) Cabeza, B) Pigmentación de aleta dorsal y anal, C) Estómago, D) Aleta pélvica, E) Inicio de alargamiento de la mandíbula, F) Detalle de la cabeza con mandíbula alargada, G) Pigmentación aleta dorsal, H) Aleta caudal. (Escala $500 \mu \mathrm{m}$ )

Fig. 2. Larval development of Hemirhamphus brasiliensis. A) Head, B) Dorsal and anal fin pigmentation, C) Gut, D) Pelvic fin, E) Start of elongated jaw, F) Head details with elongated jaw, G) Dorsal fin pigmentation, H) Caudal fin (scale $500 \mu \mathrm{m})$. 
cuerpo con escamas, tres de los radios de las aletas pélvicas eran pigmentados de amarillo y la membrana interracial de negro, las bases de las aletas dorsal y anal eran de color negro; se desarrolló el preopérculo. El día 12 se detectaron jóvenes con la proyección del labio inferior de $194.5 \pm 0.26 \mu \mathrm{m}$, aletas pélvicas totalmente de color negro. Los valores de las características morfométricas de las larvas se presentan en el cuadro 1 y las características merísticas en los cuadros 2 y 3 , considerados durante 12 días de experiencia. La experiencia finalizó a los 53 días, presentando los jóvenes una longitud total de $4.8 \mathrm{~cm}$ (Fig. 3).

\section{Algunos aspectos fisiológicos}

\section{Organogénesis}

A las 24 h, el corazón impulsaba la sangre sin pigmentación por un solo canal, a las $48 \mathrm{~h}$, la sangre se pigmentó con pequeños corpúsculos rojos, a las $72 \mathrm{~h}$ el saco vitelino se redujo en $25 \%$ y el embrión giró dentro de la membrana coriónica con suaves movimientos. Los ojos presentaron movimientos, las aletas pectorales iniciaron sus movimientos, el corazón aceleró sus pulsaciones, observándose sincronización entre las contracciones de la boca y los latidos del corazón.

\section{Desarrollo larval}

A las $24 \mathrm{~h}$ de nacidas, las larvas presentaban boca amplia y pigmentada de negro que podía atrapar y consumir microalgas, rotíferos (Brachionus plicatilis), copépodos (Apocyclops distans) y metanauplios de Artemia de $48 \mathrm{~h}$ de nacidos. El estómago era largo en donde se podían mantener hasta 30 metanauplios de Artemia.

A las $240 \mathrm{~h}$ las larvas ingirieron Metamysidosis insulares como alimento zooplanctónico y eran muy resistentes a la manipulación.

\section{DISCUSIÓN}

Los huevos del pez H. brasiliensis fueron transparentes con un diámetro de 1923.54 $\pm 72.35 \mu \mathrm{m}$ al momento del desove, este valor se encuentra en la escala $(1.70-2.32 \mathrm{~mm})$ indicada para esta especie por Richards (2006), asimismo presentaron caracteres externos con cierto parecido a los de $X$. brasiliensis pero con un número mayor de filamentos corionicos que les permitió adherirse a estructuras flotantes o rígidas (Fischer et al. 1995), con abundante volumen de vitelino para nutrir el embrión hasta el momento de nacer las larvas (Del Río et al. 2005). Internamente poseían

CUADRO 1

Valores promedio ( \pm desviación estándar) de características morfométricas ( $m$ m) de larvas de Hemirhamphus brasiliensis desde la eclosión hasta doce días de edad

TABLE 1

Average values ( \pm standard deviation) of morphometric characteristics of Hemirhamphus brasiliensis larvae from hatching to twelve days old

$\begin{array}{ccccc}\text { Días } & \text { Longitud total } & \text { Longitud notocordio } & \text { Longitud horquilla } & \text { Ancho larva } \\ 0 & 6.69 \pm 0.40 & 5.77 \pm 0.38 & & \\ 1 & 10.30 \pm 1.36 & 8.99 \pm 1.38 & & \\ 3 & 14.36 \pm 1.44 & 12.35 \pm 1.08 & & 1.24 \pm 0.37 \\ 6 & 17.35 \pm 0.94 & 15.63 \pm 0.57 & 20.43 \pm 0.15 & 2.53 \pm 0.12 \\ 9 & 22.05 \pm 0.00 & 18.67 \pm 0.29 & 29.50 \pm 2.50 & 3.09 \pm 0.45 \\ 12 & 32.00 \pm 4.00 & 27.50 \pm 2.50 & & \end{array}$


CUADRO 2

Valores promedio ( \pm desviación estándar) de características meristicas ( $\mathrm{mm}$ ) de larvas de Hemirhamphus brasiliensis desde la eclosión hasta doce días de edad

TABLE 2

Average values ( \pm standard deviation) of meristic characteristics of Hemirhamphus brasiliensis larvae from hatching to twelve days old

\begin{tabular}{|c|c|c|c|c|c|c|c|}
\hline Días & Boca ancho & Boca largo & Ojo ancho & Ojo largo & $\begin{array}{l}\text { Circulo orbital } \\
\text { ancho }\end{array}$ & $\begin{array}{c}\text { Circulo orbital } \\
\text { largo }\end{array}$ & $\begin{array}{c}\text { Fosa orbital } \\
\text { ancho }\end{array}$ \\
\hline 0 & $0.27 \pm 0.01$ & $0.29 \pm 0.01$ & $0.21 \pm 0.01$ & $0.19 \pm 0.02$ & $0.43 \pm 0.01$ & $0.38 \pm 0.02$ & $0.56 \pm 0.01$ \\
\hline 1 & $0.77 \pm 0.36$ & $0.57 \pm 0.29$ & $0.29 \pm 0.08$ & $0.24 \pm 0.04$ & $0.60 \pm 0.06$ & $0.55 \pm 0.04$ & $0.76 \pm 0.10$ \\
\hline 3 & $0.42 \pm 0.06$ & $0.48 \pm .07$ & $0.34 \pm 0.02$ & $0.31 \pm 0.01$ & $0.72 \pm 0.04$ & $0.65 \pm 0.04$ & $0.94 \pm 0.01$ \\
\hline 6 & $0.49 \pm 0.17$ & $0.51 \pm 0.16$ & $0.48 \pm 0.00$ & $0.44 \pm 0.03$ & $0.91 \pm 0.06$ & $0.82 \pm 0.05$ & $1.12 \pm 0.07$ \\
\hline 9 & $0.42 \pm 0.00$ & $0.44 \pm 0.04$ & $0.49 \pm 0.00$ & $0.43 \pm 0.05$ & $1.06 \pm 0.02$ & $0.95 \pm 0.10$ & $1.33 \pm 0.00$ \\
\hline 12 & $0.77 \pm 0.01$ & $0.88 \pm 0.31$ & $0.73 \pm 0.12$ & $0.75 \pm 0.20$ & $1.60 \pm 0.09$ & $1.42 \pm 0.26$ & $1.94 \pm 0.08$ \\
\hline Días & $\begin{array}{c}\text { Fosa orbital } \\
\text { largo }\end{array}$ & $\begin{array}{l}\text { Longitud } \\
\text { cabeza }\end{array}$ & $\begin{array}{c}\text { Longitud } \\
\text { preanal }\end{array}$ & $\begin{array}{c}\text { Longitud } \\
\text { postanal }\end{array}$ & *LLS & *LLI & *Radios \\
\hline 0 & $0.50 \pm 0.01$ & $1.51 \pm 0.06$ & $3.03 \pm 0.98$ & $2.08 \pm 0.12$ & & & $16 \pm 0$ \\
\hline 1 & $0.68 \pm 0.07$ & $2.45 \pm 0.34$ & $8.13 \pm 1.57$ & $3.30 \pm 1.47$ & & & $16 \pm 0$ \\
\hline 3 & $0.84 \pm 0.06$ & $3.18 \pm 0.11$ & $9.16 \pm 0.85$ & $5.19 \pm 0.60$ & & & $16 \pm 1$ \\
\hline 6 & $1.01 \pm 0.02$ & $3.81 \pm 0.19$ & $11.96 \pm 0.66$ & $5.39 \pm 0.28$ & $1.08 \pm 0.25$ & $1.23 \pm 0.35$ & $19 \pm 1$ \\
\hline 9 & $1.21 \pm 0.01$ & $3.29 \pm 1.11$ & $14.55 \pm 0.15$ & $7.50 \pm 0.15$ & $0.67 \pm 0.09$ & $1.39 \pm 0.44$ & $20 \pm 0$ \\
\hline 12 & $1.69 \pm 0.32$ & $6.87 \pm 0.84$ & $20.50 \pm 2.50$ & $11.50 \pm 1.50$ & $1.33 \pm 0.72$ & $2.36 \pm 2.36$ & $20 \pm 1$ \\
\hline
\end{tabular}

LLS = longitud del lóbulo superior, LLI = longitud del lóbulo inferior.

*Medidas de la aleta caudal.

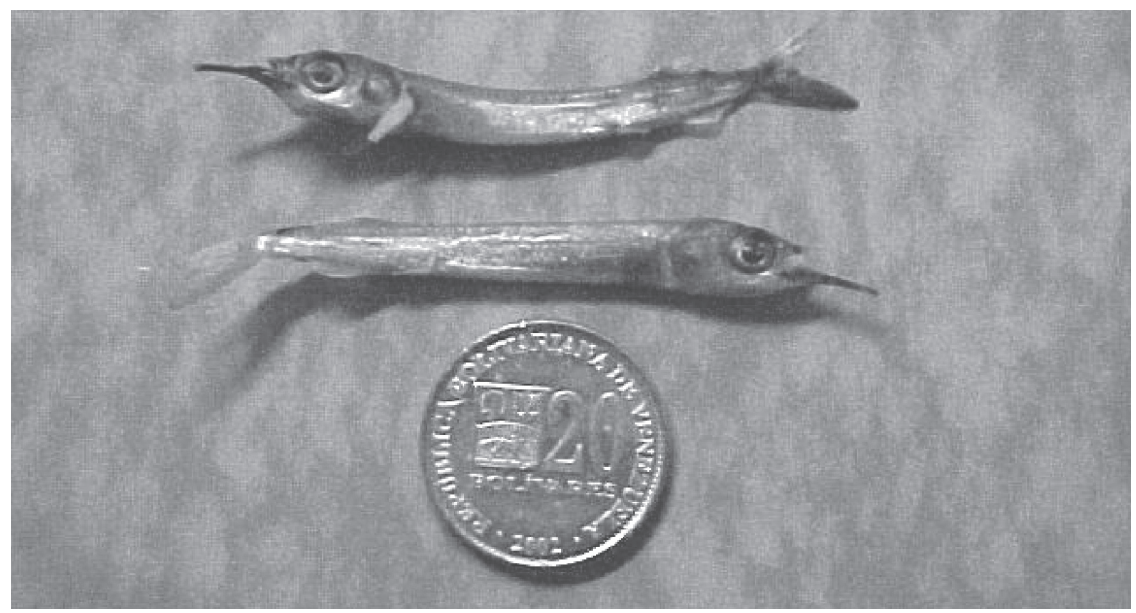

Fig. 3. Jóvenes de Hemirhamphus brasiliensis de 53 días de edad, completamente transformados con mandíbula inferior alargada. Escala: $20 \mathrm{~mm}$.

Fig. 3. Young of Hemirhamphus brasiliensis aged 53 days, totally transformed with inferior elongated jaw. Scale: $20 \mathrm{~mm}$. 


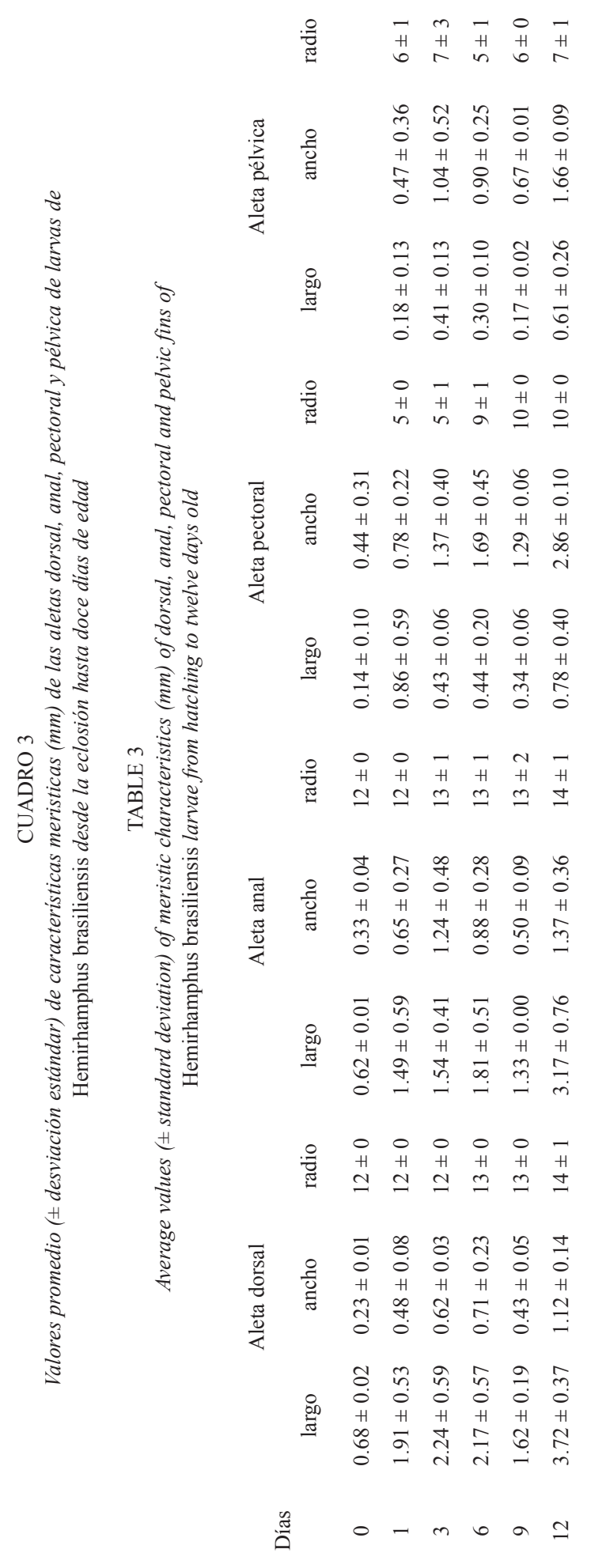


una sola gota lipídica como en otras especies de peces marinos (Cuartas et al. 2004, Querales et al. 2004).

El desarrollo embrionario se completó en $112 \mathrm{~h}$, desarrollándose toda la organogénesis: corazón, hígado, branquias y vesícula biliar como ocurrió en el desarrollo embrionario de $X$. brasiliensis (Del Río et al. 2005), al nacer las larvas en forma de torpedo presentaron pigmentación verde-amarillo, al respecto Cervigón (1980) refiere que $H$. brasiliensis se distingue de $H$. balao por sus caracteres morfológicos y merísticos y por la coloración que en el primero es amarillo-naranja y en el segundo es rosado.

Los numerosos melanóforos puntiformes y dendriformes en toda la región del cuerpo, así como los caracteres morfométricos y merísticos son considerados como elementos taxonómicos en la identificación de larvas en peces marinos (Marín et al. 1995, Balza et al. 2001). En el caso de estas larvas, no solo deben realizar una metamorfosis para su identificación, sino que deben desarrollar la mandíbula inferior que se proyecta en forma de pico prolongado con la terminación redondeada de color rojo (Cervigón 1980).

Las larvas recién eclosionadas tuvieron una longitud total de $6.69 \pm 0.40 \mathrm{~mm}$, valor que se encuentra en la escala $(5-7 \mathrm{~mm})$ indicada para la especie por Richards (2006), otras comparaciones con el mismo autor y que concuerdan con los valores obtenidos en este trabajo son las relacionadas con el número de radios en la aleta dorsal (12-14) muy cercano al obtenido por Richards (2006) de 12 a 15 radios, en la aleta anal se cuantificó de 12 a 14 radios mientras que Richards (2006) menciona de 11 a 15 radios y más frecuentemente en número de 13 , para la aleta pélvica coincide el número de radios (6) con el indicado por Richards (2006). Otras comparaciones descriptivas, morfológicas y merísticas de esta especie con otras del mismo género no fueron posibles debido a la no disponibilidad de bibliografía con información sobre desarrollo embrionario y larval de esta $u$ otras especies de la familia Hemirhamphidae.
Algunos aspectos fisiológicos: la forma de torpedo que presentaron las larvas de $H$. brasiliensis al momento de la eclosión, les permitió impulsarse vigorosamente. Con capacidad de atrapar y digerir rotíferos (Brachionus plicatilis) y copépodos (Apocyclops distans), no así a las microalgas que también logró consumir pero que fueron observadas sin digerir en el tracto digestivo de larvas sacrificadas. Cabrera et al. (1997) señalan que las larvas de hábito alimenticio del tipo carnívoro no pueden digerir las microalgas por su incapacidad de metabolizarlas, debido a la constitución de su pared celular (Cupp 1977).

La alta capacidad para ingestar zooplancton fue debido a lo largo del tubo digestivo en donde se pudo cuantificar hasta 30 metanauplios de Artemia de 48 h. de eclosionados. En los peces teleósteos el alargamiento del tracto digestivo es una estrategia para aumentar la capacidad de absorción y poder así obtener los diferentes nutrientes. En otras especies el intestino es un tubo recto que luego se torna curvo en la medida de su desarrollo (Gisbert 1998).

Se concluye que los huevos del pez Hemirhamphus brasiliensis eran de 1923.54 $\pm 72.35 \mu \mathrm{m}$, con superficie estriada y numerosos filamentos coriónicos, que les permitía adherirse a objetos flotantes. En el embrión se desarrolló toda su organogénesis, naciendo una larva en forma de torpedo muy resistente a la manipulación que digirió zooplancton más no microalgas y alcanzó la etapa de joven en nueve días, desarrollando la mandíbula inferior en forma de pico al metamorfosearse a los 12 días de nacidas.

\section{RESUMEN}

Se describe la formación del embrión y el desarrollo larval del pez Hemirhamphus brasiliensis Linnaeus, 1758, a partir de huevos en estado de mórula, recolectados en el alga parda Sargassum sp. Los huevos eran esféricos con un diámetro de $1923.54 \pm 72.35 \mu \mathrm{m}$, con numerosos filamentos coriónicos y estrías en su superficie. Durante las primeras $48 \mathrm{~h}$, el embrión desarrolló la vesícula cefálica, los miomeros y el corazón, el cual se ubicó en el exterior de cuerpo impulsando sangre incolora, la cual se pigmentó de rojo posteriormente. Antes de la eclosión se desarrollaron el riñón, estómago, hígado y la vesícula biliar, las aletas 
pectorales, cuatro pares de arcos branquiales y la boca. Las larvas eclosionaron a la $114 \mathrm{~h}$, presentando el cuerpo robusto en forma de torpedo, verde-amarillo con melanoforos dendriformes. Al nacer ingirieron metanauplios de Artemia. A las 72 h después de la eclosión se observó el esbozo de la aleta pélvica y a las 240 h se completó la metamorfosis.

Palabras clave: embrión, eclosión, Hemirhamphus brasiliensis, larva.

\section{REFERENCIAS}

Ahlstrom, E. 1968. Review of Development of fishes of the Chesapeake Bay Region, an atlas of egg, larval and juveniles stages. Copeia 1: 648-651.

Balza, M., M. Gutierrez \& B. Marín. 2001. Descripción morfológica y crecimiento en los primeros estadios larvarios de la sardina Sardinella aurita (Valenciennes, 1847) (Pisces: Clupeidae). Bol. Inst. Oceanog. Univ. Venezuela 40: 91-101.

Cabrera, T., J. Rosas \& J. Millán. 1997. Reproducción y Desarrollo Larvario del Pargo Dientón, Lutjanus griseus en cautiverio. Rev. Carib. Jour. Sci. 33: 239245 .

Castro, J.J., J. Santiago \& L. Santana-Ortega. 2002. A general theory on fish aggregation to floating objects: an alternative to the meeting point hypothesis. Rev. Fish Biol. Fisheries 11: 255-277.

Cervigón, F. 1980. Ictiología Marina. Fundación Científica Los Roques, Caracas, Venezuela.

Cervigón, F. 1991. Los peces marinos de Venezuela. Fundación Científica Los Roques, Caracas, Venezuela.

Cuartas, A., J. Rosas, A. Velásquez \& T. Cabrera. 2004. Inducción al desove, desarrollo embrionario y larval del Corocoro rayao Haemulon bonariense Cuvier,1830 (Pisces: Haemulidae). Rev. Mar Biol. Oceanogr. 38: 27-37.

Cupp, E. 1977. Marine plankton diatoms of the west coast of North America. Bull. Scripp. Inst. Oceanog. Tech. Ser. 5: 1-238.

Del Río, V., J. Rosas, A. Velásquez \& T. Cabrera. 2005. Desarrollo embrionario-larval y tiempo de metamorfosis del pez tropical Xenomelaniris brasiliensis (Pisces: Atherinidae). Rev. Biol. Trop. 53: 503-513.

Fischer, W., F. Krupp, W. Schneider, C. Sommer, K.E. Carpenter \& V.H. Niem. 1995. Guía FAO para la identificación de especies para los fines de pesca. Pacífico centro-oriental. Roma 2: 1091-1095.

Gisbert, E., A. Rodríguez, F. Castello-Orvay \& P. Williot. 1998. A histological study of the development of the digestive tract of Siberian sturgeon (Acipenser baeri) during early ontogeny. Aquaculture 167: 195-209.

Marín, B., O. Díaz \& R. Briceño. 1995. Aspectos descriptivos de los ovocitos y postlarvas de tinícalo, Xenomelaniris brasiliensis (Quoy y Gaimard) (Pisces: Atherinidae). Bol. Inst. Oceanog. Venezuela 34: 59-68.

McBride, R.S. \& P.E. Thurman. 2003. Reproductive Biology of Hemiramphus brasiliensis and H. balao (Hemiramphidae): Maturation, Spawning Frequency, and Fecundity. Biol. Bull. 204: 57-67.

Querales, D., J. Rosas, A. Velásquez, T. Cabrera \& C. Maneiro. 2004. Desarrollo embrionario y larval de Paralabrax dewegeri Metzelaar, 1919 (Pisces:Serranidae). Rev. Biol. Mar. Oceangr. 39: $1-11$.

Richards, W.J. 2006. Early stages of Atlantic fishes: An identification guide for the Western Central North Atlantic. CRC, Florida, EEUU. 